\title{
THE INTEGRAL EQUATION METHODS FOR THE PERTURBED HELMHOLTZ EIGENVALUE PROBLEMS
}

\author{
ABDESSATAR KHELIFI
}

Received 10 May 2004 and in revised form 31 January 2005

It is well known that the main difficulty in solving eigenvalue problems under shape deformation relates to the continuation of multiple eigenvalues of the unperturbed configuration. These eigenvalues may evolve, under shape deformation, as separated, distinct eigenvalues. In this paper, we address the integral equation method in the evaluation of eigenfunctions and the corresponding eigenvalues of the two-dimensional Laplacian operator under boundary variations of the domain. Using surface potentials, we show that the eigenvalues are the characteristic values of meromorphic operator-valued functions.

\section{Introduction}

The properties of eigenvalue problems under shape deformation have been the subject of comprehensive studies $[7,13]$ and the area continues to carry great importance up to now [14]. A substantial portion of these investigations is related to smoothness properties of eigenfunctions with respect to boundary perturbations. Recently, Bruno and Reitich have presented in [4] explicit constructions of high-order boundary perturbation expansions for eigenelements. Their algorithm is based on certain properties of joint analytic dependence on the boundary perturbations and spatial variables of the eigenfunctions. The main difficulty in solving eigenvalue problems relates to the continuation of multiple eigenvalues of the unperturbed configuration. These eigenvalues may evolve, under shape deformation, as separated, distinct eigenvalues, and this splitting may only become apparent at high orders in their Taylor expansions.

In this paper, we use the technique of integral equation to evaluate the analyticity properties and asymptotic expansions of the eigenfunctions and the eigenvalues of the Laplacian operator under boundary variations of the domain of definition. Using surface potentials, we show that the eigenvalues are the characteristic values of meromorphic operator-valued functions which are of Fredholm type with index 0. We then proceed using the generalized Rouchés theorem [6] and the result found in [10] to construct their complete asymptotic expressions. Our approach concerning the question of analytic dependence and asymptotic expansion is based on a holomorphic formulation of the boundary integral equations and its characteristic problem version. 
Our analysis and uniform asymptotic formulas of the eigenfunctions, which are represented as sum of a single-layer potential and of a double-layer potential involving the Green's function, are considerably different from that in [9]. We would here like to find an efficient and accurate method, different from what we have presented in [2]. Powerful techniques from the theory of meromorphic operator-valued functions and careful asymptotic analysis of integral kernels are combined for solving this problem. Similar results can be obtained when considering the homogeneous Neumann boundary condition with only minor modifications of the arguments and techniques presented in this work. We just replace the Dirichlet Green's function by the Neumann Green's function in the integral representation and in a way completely similar, we establish our asymptotic formulas.

\section{Problem formulation}

Let $\gamma(t):[0,1] \rightarrow \mathbb{R}^{2}$ and $\beta(t):[0,1] \rightarrow \mathbb{R}^{2}$ be two analytic, 1-periodic functions satisfying $\left|\gamma^{\prime}(t)\right|>C$ for all $t \in[0,1]$, where $C$ is a positive constant. We introduce

$$
\gamma_{\epsilon}(t)=\gamma(t)+\epsilon \beta(t), \quad \epsilon \in \mathbb{R}
$$

With this definition, $(t, \epsilon) \mapsto \gamma_{\epsilon}(t)$ is an analytic function on $[0,1] \times \mathbb{R}, 1$-periodic in the variable $t$, and satisfies

$$
\left|\gamma_{\epsilon}^{\prime}(t)\right|>\frac{C}{2}
$$

for any $\epsilon$ such that $2|\epsilon|\left|\beta^{\prime}(t)\right|_{\mathscr{C}^{0}([0,1])}<C$. We consider the bounded domain $\Omega_{\epsilon}$ in $\mathbb{R}^{2}$ with boundary $\partial \Omega_{\epsilon}$ parameterized by the function $\gamma_{\epsilon}(t)$ :

$$
\partial \Omega_{\epsilon}=\left\{\gamma_{\epsilon}(t), t \in[0,1]\right\}
$$

The outward unit normal to $\partial \Omega_{\epsilon}$ is denoted by $v_{\epsilon}$.

In this paper, we deal with the asymptotics of eigenvalues and eigenfunctions associated to the following eigenvalue problem:

$$
\Delta u(\epsilon)+\lambda^{2}(\epsilon) u(\epsilon)=0 \quad \text { in } \Omega_{\epsilon}, \quad u(\epsilon)=0 \quad \text { on } \partial \Omega_{\epsilon} .
$$

It is well known that the operator $-\Delta$ on $L^{2}\left(\Omega_{\epsilon}\right)$ with domain $H^{2}\left(\Omega_{\epsilon}\right) \cap H_{0}^{1}\left(\Omega_{\epsilon}\right)$ is selfadjoint with compact resolvent. Consequently, its spectrum only consists of isolated, real, and positive eigenvalues with finite multiplicity, and there are corresponding eigenfunctions which build an orthonormal basis of $L^{2}\left(\Omega_{\epsilon}\right)$.

Let $\lambda_{0}^{2}>0$ denote an eigenvalue of the eigenvalue problem (2.4) for $\epsilon=0$ with geometric multiplicity $m \geq 1$. There exists a small constant $r_{0}>0$ such that $\lambda_{0}^{2}$ is the unique eigenvalue of (2.4) for $\epsilon=0$ in the set $\left\{\lambda^{2}, \lambda \in D_{r_{0}}\left(\lambda_{0}\right)\right\}$, where $D_{r_{0}}\left(\lambda_{0}\right)$ is a disc of center $\lambda_{0}$ and radius $r_{0}$. We define the $\lambda_{0}$-group as the whole perturbed eigenvalues of (2.4) for $\epsilon>0$ generated by splitting from $\lambda_{0}$. The following analyticity result is well known [7].

THeorem 2.1. There exists $\epsilon_{0}>0$ such that for $|\epsilon|<\epsilon_{0}$, the $\lambda_{0}$-group consists of m eigenvalues, $\lambda_{i}(\epsilon), i=1, \ldots, m$ (repeated according to their multiplicity). Moreover, these $\lambda_{i}(\epsilon)$ are 
analytic functions with respect to $\epsilon$ satisfying $\lambda_{i}(0)=\lambda_{0}, i=1, \ldots, m$. The normalized eigenfunctions associated to the $\lambda_{0}$-group of eigenvalues are analytic with respect to $\epsilon$ and their values at 0 are $m$ linearly independent solutions of the unperturbed eigenvalue problem.

Classical regularity results and the previous theorem imply that the eigenfunctions associated to the $\lambda_{0}$-group of eigenvalues are separately analytic in the small parameter $\epsilon$ and the spatial variable $x$. Using an integral equation technique, we will also establish the joint analytic dependence of these functions with respect to $(x, \epsilon)$. As it is well known, joint analyticity does not follow from separate real analyticity properties.

\section{Integral equation method}

The use of integral equations is a convenient tool for a number of investigations [5]. We now develop a boundary integral formulation for solving the eigenvalue problem (2.4). We use this method to characterize the eigenvalue and the normal derivative of the eigenfunction as characteristic value and root function of some operator-valued function. This characterization is the key point in deriving our results and asymptotic formulae.

Let $u$ be the solution to the Helmholtz equation:

$$
\Delta u+\lambda^{2} u=0, \quad \text { in } \mathbb{R}^{2} .
$$

We begin by defining the outgoing Green's function $G(x, y)$ as the solution of

$$
\Delta_{x} G(x, y)+\lambda^{2} G(x, y)=-\delta_{y}(x), \quad \text { in } \mathbb{R}^{2},
$$

with the radiation condition as $|x| \rightarrow+\infty$ :

$$
\left|\frac{\partial G}{\partial|x|}-i \lambda G\right|=O\left(\frac{1}{|x|}\right)
$$

In fact, $G$ is explicitly given as

$$
G(x, y)=\frac{i}{4} H_{0}^{(1)}(\lambda|x-y|)
$$

where $H_{0}^{(1)}(z)$ is the Hankel function of the first kind and of order zero [1]. Its singularity has the form

$$
G(x, y) \sim \frac{1}{2 \pi} \log |x-y|+\cdots \quad \text { as } x \longrightarrow y .
$$

3.1. Preliminary results. Consider (3.1) for the function $u$ in the exterior of $\Omega_{\epsilon}$, multiply by the Green's function $G$ and integrate by parts, we get that for $x \in \mathbb{R}^{2} \backslash \overline{\Omega_{\epsilon}}$,

$$
u(x)=\int_{\partial \Omega_{\epsilon}}\left(\left.\frac{\partial u}{\partial v_{\epsilon}}\right|_{+}(y) G(x, y)-\left.u(y) \frac{\partial G}{\partial \nu_{\epsilon}(y)}\right|_{+}(x, y)\right) d \sigma(y) .
$$

The jump condition for $\partial u / \partial v_{\epsilon}$ on $\partial \Omega_{\epsilon}$ yields

$$
u(x)=-\left.\int_{\partial \Omega_{\epsilon}} \frac{\partial G}{\partial \nu_{\epsilon}(y)}\right|_{+}(x, y) u(y) d \sigma(y)+\left.\int_{\partial \Omega_{\epsilon}} G(x, y) \frac{\partial u}{\partial \nu_{\epsilon}}\right|_{-}(y) d \sigma(y) .
$$


Of course, the above equations does not hold up to the boundary of $\Omega_{\epsilon}$, but if we take the limit as $x \rightarrow \partial \Omega_{\epsilon}$, we get from, for instance, $[5,12]$ that

$$
\begin{aligned}
\left.\frac{1}{2} u\right|_{\partial \Omega_{\epsilon}}(x)= & -\left.\int_{\partial \Omega_{\epsilon}} \frac{\partial G}{\partial v_{\epsilon}(y)}\right|_{+}(x, y) u(y) d \sigma(y) \\
& +\left.\int_{\partial \Omega_{\epsilon}} G(x, y) \frac{\partial u}{\partial \nu_{\epsilon}}\right|_{-}(y) d \sigma(y) \text { for } x \in \partial \Omega_{\epsilon} .
\end{aligned}
$$

We introduce the following operators, called single- and double-layer potentials, respectively:

$$
\begin{aligned}
& \operatorname{Sl}(\lambda) g(x)=\int_{\partial \Omega_{\epsilon}} G(x, y) g(y) d \sigma(y), \quad x \in \mathbb{R}^{2} \backslash \partial \Omega_{\epsilon}, \\
& \operatorname{Dl}(\lambda) g(x)=\int_{\partial \Omega_{\epsilon}} \partial_{\nu_{\epsilon}(y)} G(x, y) g(y) d \sigma(y), \quad x \in \mathbb{R}^{2} \backslash \partial \Omega_{\epsilon} .
\end{aligned}
$$

For $g \in \mathscr{C}^{\infty}\left(\partial \Omega_{\epsilon}\right)$, or even $g \in L^{1}\left(\partial \Omega_{\epsilon}\right)$, the functions $\operatorname{Sl}(\lambda) g$ and $\mathrm{Dl}(\lambda) g$ are well defined and smooth for $x \in \mathbb{R}^{2} \backslash \partial \Omega_{\epsilon}$.

Now define the following operators:

$$
S(\lambda): H^{-1 / 2}\left(\partial \Omega_{\epsilon}\right) \longrightarrow H^{1 / 2}\left(\partial \Omega_{\epsilon}\right), \quad D(\lambda): H^{1 / 2}\left(\partial \Omega_{\epsilon}\right) \longrightarrow H^{1 / 2}\left(\partial \Omega_{\epsilon}\right),
$$

where

$$
\begin{aligned}
& S(\lambda): g \longrightarrow \int_{\partial \Omega_{\epsilon}} G(\cdot, y) g(y) d \sigma(y), \\
& D(\lambda):\left.g \longrightarrow \int_{\partial \Omega_{\epsilon}} \frac{\partial G}{\partial \nu_{\epsilon}(y)}\right|_{+}(\cdot, y) g(y) d \sigma(y) .
\end{aligned}
$$

For such $g$ and every $x \in \partial \Omega_{\epsilon}$, we denote by $g_{+}(x)$ and $g_{-}(x)$ the limits of $g(y)$ as $y \rightarrow x$, from $y \in \Omega_{\epsilon}$ and $y \in \mathbb{R}^{2} \backslash \overline{\Omega_{\epsilon}}$, respectively, when these limits exist. It is a wellknown classical result that, for $x \in \partial \Omega_{\epsilon}$,

$$
\begin{aligned}
& (\mathrm{Sl}(\lambda) g)_{+}(x)=(\operatorname{Sl}(\lambda) g)_{-}(x)=S(\lambda) g(x), \\
& (\operatorname{Dl}(\lambda) g)_{ \pm}(x)= \pm \frac{1}{2} g(x)+D(\lambda) g(x),
\end{aligned}
$$

where $S(\lambda)$ is pseudodifferential operator of order -1 .

Throughout this paper, we use for simplicity the notation $H_{\#}^{s}(] 0,1[)=H^{s}(\mathbb{R} /] 0,1[)$, for $s \in \mathbb{R}$, where $H^{s}(\mathbb{R} /] 0,1[)$ denotes the classical Sobolev $H^{s}$-space on the quotient $\mathbb{R} /] 0,1[$.

Using a change of variables and integral equations, the following important result immediately holds from Taylor [16, page 184].

Proposition 3.1. Let $L_{\epsilon}(\lambda): H_{\#}^{-1 / 2}(] 0,1[) \rightarrow H_{\#}^{1 / 2}(] 0,1[)$ be defined as follows:

$$
\begin{aligned}
L_{\epsilon}(\lambda) f(t) & =\left(S(\lambda) f\left(\gamma_{\epsilon}^{-1}\right)\right)\left(\gamma_{\epsilon}(t)\right) \\
& =\frac{i}{4} \int_{0}^{1} H_{0}^{(1)}\left(\lambda\left|\gamma_{\epsilon}(t)-\gamma_{\epsilon}(s)\right|\right)\left|\gamma_{\epsilon}^{\prime}(s)\right| f(s) d s \quad \text { for } f \in H_{\#}^{-1 / 2}(] 0,1[) .
\end{aligned}
$$


Then the operator-valued function $L_{\epsilon}(\lambda)$ is Fredholm analytic with index 0 in $\mathbb{C} \backslash i \mathbb{R}^{-}$. Moreover, $L_{\epsilon}^{-1}(\lambda)$ is a meromorphic function and its poles are in $\{\mathfrak{J}(z) \leq 0\}$. $\mathfrak{J}(z)$ means the imaginary part of $z$ and $\mathfrak{R}(z)$ is the real part.

From now on we will focus our attention on solving the eigenvalue problem (2.4).

3.2. Joint analyticity of kernel. Based on the result found in [3] and on the argument of Millar [11], we will now prove the following proposition. The following result will be useful in Sections 4 and 5.

Proposition 3.2. There exist a constant $\eta>0$ and a complex neighborhood $\mathscr{V}$ of 0 such that for every function $\varphi(t, \epsilon) \in H_{\#}^{-1 / 2}(] 0,1[)$ analytic in $(t, \epsilon) \in\{|\mathfrak{J}(t)| \leq \eta\} \times \mathscr{V}$, the function $L_{\epsilon}(\lambda) \varphi(t, \epsilon) \in H_{\#}^{1 / 2}(] 0,1[)$ is analytic with respect to $(t, \epsilon, \lambda)$ in $\{|\mathfrak{J}(t)| \leq \eta\} \times \mathscr{V} \times D_{r_{0}}\left(\lambda_{0}\right)$, where $D_{r_{0}}\left(\lambda_{0}\right)$ is a disc of center $\lambda_{0}$ and radius $r_{0}$.

Proof. The proof of this proposition heavily relies on the work of Bruno and Reitich [3] where they expressed the kernel of $L_{\epsilon}(\lambda)$ in the following form:

$$
\begin{aligned}
& \frac{i}{4} H_{0}^{(1)}\left(\lambda\left|\gamma_{\epsilon}(t)-\gamma_{\epsilon}(s)\right|\right)\left|\gamma_{\epsilon}^{\prime}(s)\right| \\
& \quad=-\sum_{p=-1}^{1} \log (|t-s-p|) h\left(\lambda^{2}\left|\gamma_{\epsilon}(t)-\gamma_{\epsilon}(s)\right|^{2}\right)\left|\gamma_{\epsilon}^{\prime}(s)\right|+k(t, s, \epsilon, \lambda),
\end{aligned}
$$

for $(t, s, \epsilon, \lambda) \in \mathscr{J} \cap\{\mathfrak{J}(t)=0\} \cap\{\mathfrak{J}(s)=0\} \cap\{\mathfrak{J}(\epsilon)=0\}, s \notin\{t, t+1, t-1\}$, where $h$ is an analytic function in $\mathbb{C}$, and $k$ is analytic in $\mathscr{J}$. Here $\mathscr{J}=\{|\mathfrak{J}(t)| \leq \eta ;|\mathfrak{J}(s)| \leq \eta ;|\epsilon| \leq$ $\left.\delta ; \lambda \in D_{r_{0}}\left(\lambda_{0}\right) ;-\rho \leq \mathfrak{R}(t) \leq 1+\rho ;-\rho \leq \mathfrak{R}(s) \leq 1+\rho\right\}$, for the positive numbers $\rho, r_{0}, \eta$, and $\delta$.

The central difficulty to prove the analytic property of the operator $L_{\epsilon}$ comes from the spatial regularity of its kernel. We show that logarithmic singularity of the kernel of $L_{\epsilon}(\lambda)$ is independent of the parameter $\epsilon$.

We introduce

$$
\Phi(t, \epsilon, \lambda)=-\int_{0}^{1} \sum_{p=-1}^{1} \log |t-s-p| h\left(\lambda^{2}\left|\gamma_{\epsilon}(t)-\gamma_{\epsilon}(s)\right|^{2}\right) \varphi(s, \epsilon)\left|\gamma_{\epsilon}^{\prime}(s)\right| d s
$$

Using a change of variables, this function can be rewritten as follows:

$$
\Phi(t, \epsilon, \lambda)=-\int_{-1}^{2} \log |t-s| h\left(\lambda^{2}\left|\gamma_{\epsilon}(t)-\gamma_{\epsilon}(s)\right|^{2}\right) \varphi(s, \epsilon)\left|\gamma_{\epsilon}^{\prime}(s)\right| d s
$$

An integration by parts leads to

$$
\begin{aligned}
\Phi(t, \epsilon, \lambda)= & \log (|t-2|) \psi(t, 2, \epsilon, \lambda) \\
& -\log (|t+1|) \psi(t,-1, \epsilon, \lambda)-\int_{-1}^{2} \frac{\psi(t, s, \epsilon, \lambda)}{|t-s|} d s
\end{aligned}
$$


where

$$
\psi(t, s, \epsilon, \lambda)=-\int_{t}^{s} h\left(\lambda^{2}\left|\gamma_{\epsilon}(t)-\gamma_{\epsilon}(z)\right|^{2}\right) \varphi(z, \epsilon)\left|\gamma_{\epsilon}^{\prime}(z)\right| d z
$$

Clearly, $\Phi(t, \epsilon, \lambda)$ can be extended to a complex analytic function in $\mathbb{C} \times \mathscr{V} \times D_{r_{0}}\left(\lambda_{0}\right)$. Furthermore, from the following identity

$$
L_{\epsilon}(\lambda) \varphi(t, \epsilon)=\Phi(t, \epsilon, \lambda)+\int_{0}^{1} k(t, s, \epsilon, \lambda) \varphi(s, \epsilon) d s,
$$

we deduce the desired result applying (3.14).

\section{Reduction to a characteristic value problem}

In this section, we first recall some definitions and notations from $[6,10]$. Using boundary integral equations, we reduce the problem (2.4) to the existence and the distribution of the characteristic values of selfadjoint integral operators in the complex plane.

4.1. Notations and definitions. Let $\mathscr{E}$ and $\mathscr{F}$ be two Banach spaces and let $\mathscr{L}(\mathscr{E}, \mathscr{F})$ be the algebra of all bounded-valued function acting from $\mathscr{E}$ into $\mathscr{F} \mathscr{E}^{\prime}$ and $\mathscr{F}^{\prime}$ denote the dual spaces of $\mathscr{E}$ and $\mathscr{F}$, respectively.

Let $\lambda_{0}$ be a fixed complex value in $\mathbb{C}$. We denote by $\mathscr{A}(\lambda)$ an operator-valued function acting from $D_{r_{0}}\left(\lambda_{0}\right)$ into $\mathscr{L}(\mathscr{E}, \mathscr{F})$, where $D_{r_{0}}\left(\lambda_{0}\right)$ is a disc of center $\lambda_{0}$ and radius $r_{0}>0$. $\lambda_{0}$ is called a characteristic value of $\mathscr{A}(\lambda)$ if

(i) $\mathscr{A}(\lambda)$ is holomorphic in some neighborhood of $\lambda_{0}$, except possibly at $\lambda_{0}$,

(ii) there exists a vector-valued function $\phi(\lambda): D_{r_{0}}\left(\lambda_{0}\right) \rightarrow \mathscr{H}$ holomorphic at $\lambda_{0}$ and verifying $\phi\left(\lambda_{0}\right) \neq 0$, such that $\mathscr{A}(\lambda) \phi(\lambda)$ is holomorphic at $\lambda_{0}$ and vanishes at this point.

$\phi(\lambda)$ is called a root function of $\mathscr{A}(\lambda)$ associated to $\lambda_{0}$ and the vector $\phi_{0}=\phi\left(\lambda_{0}\right)$ is called an eigenvector. The closure of the linear set of eigenvectors corresponding to $\lambda_{0}$ is denoted by $\operatorname{Ker} \mathscr{A}\left(\lambda_{0}\right)$.

Suppose that $\lambda_{0}$ is a characteristic value of the function $\mathscr{A}(\lambda)$ and $\phi(\lambda)$ is a root function satisfying (ii). Then there exists a number $m(\phi) \geq 1$ and a vector-valued function $\psi(\lambda): D_{r_{0}}\left(\lambda_{0}\right) \rightarrow \mathscr{H}$ holomorphic such that

$$
\mathscr{A}(\lambda) \phi(\lambda)=\left(\lambda-\lambda_{0}\right)^{m(\phi)} \psi(\lambda), \quad \psi\left(\lambda_{0}\right) \neq 0 .
$$

The number $m(\phi)$ is called the multiplicity of the root function $\phi(\lambda)$. Let $\phi_{0}$ be an eigenvector corresponding to $\lambda_{0}$ and let

$$
\mathscr{R}\left(\phi_{0}\right)=\left\{m(\phi) ; \phi(\lambda) \text { is a root function such } \phi\left(\lambda_{0}\right)=\phi_{0}\right\} .
$$

Then by rank of $\phi_{0}$ we mean $\operatorname{rank}\left(\phi_{0}\right)=\max \mathscr{R}\left(\phi_{0}\right)$. Suppose that $n=\operatorname{dim} \operatorname{Ker} \mathscr{A}\left(\lambda_{0}\right)<$ $+\infty$ and that the ranks of all vectors in $\operatorname{Ker} \mathscr{A}\left(\lambda_{0}\right)$ are finite. A system of eigenvectors $\phi_{0}^{j}$, $j=1, \ldots, n$, is called a canonical system of eigenvectors of $\mathscr{A}(\lambda)$ associated to $\lambda_{0}$ if the ranks possess the following property: $\operatorname{rank}\left(\phi_{0}^{j}\right)$ is the maximum of the ranks of all eigenvectors 
in some direct complement in $\operatorname{dim} \operatorname{Ker} \mathscr{A}\left(\lambda_{0}\right)$ of the linear span of the vectors $\phi_{0}^{1}, \ldots, \phi_{0}^{j-1}$. Let $r_{j}=\operatorname{rank}\left(\phi_{0}^{j}\right)$. Then $\left(r_{j}\right)_{j}$ uniquely determines the function $\mathscr{A}(\lambda)$. We call

$$
N\left(\mathscr{A}\left(\lambda_{0}\right)\right)=\sum_{j=1}^{n} r_{j}
$$

the null multiplicity of the characteristic value $\lambda_{0}$ of $\mathscr{A}(\lambda)$.

If $\lambda_{0}$ is not a characteristic value of $\mathscr{A}(\lambda)$, we put $N\left(\mathscr{A}\left(\lambda_{0}\right)\right)=0$.

Suppose that $\mathscr{A}^{-1}(\lambda)$ exists and is holomorphic in some neighborhood of $\lambda_{0}$, except possibly at $\lambda_{0}$. Then the number

$$
M\left(\mathscr{A}\left(\lambda_{0}\right)\right)=N\left(\mathscr{A}\left(\lambda_{0}\right)\right)-N\left(\mathscr{A}^{-1}\left(\lambda_{0}\right)\right)
$$

is called the multiplicity of the characteristic value $\lambda_{0}$ of $\mathscr{A}(\lambda)$. Suppose that $\lambda_{1}$ is a pole of the operator-valued function. The Laurent expansion of $\mathscr{A}(\lambda)$ in $\lambda_{1}$ is given by

$$
\mathscr{A}(\lambda)=\sum_{j \geq-s}\left(\lambda-\lambda_{1}\right)^{j} A_{j}
$$

If in the last expression the operators $A_{-j}, j=1, \ldots, s$, are finite dimensional, then $\mathscr{A}(\lambda)$ is called finitely meromorphic at $\lambda_{1}$.

The operator-valued function $\mathscr{A}(\lambda)$ is said to be of Fredholm type at the point $\lambda_{1}$ if the operator $A_{0}$ in the last expansion is a Fredholm operator.

If $\mathscr{A}(\lambda)$ is holomorphic at the point $\lambda_{0}$ and the operator $\mathscr{A}\left(\lambda_{0}\right)$ is invertible, then $\lambda_{0}$ is called a regular point of $\mathscr{A}(\lambda)$.

We set

$$
\begin{array}{ll}
(y \otimes v)(w):=(w, v) y & (w \in \mathscr{F}), \\
(v \otimes y)(u):=(y, u) v & \left(u \in \mathscr{E}^{\prime}\right)
\end{array}
$$

for $y \in \mathscr{E}$ and $v \in \mathscr{F}^{\prime}$. Note that $y \otimes v \in \mathscr{L}(\mathscr{F}, \mathscr{E}), v \otimes y \in \mathscr{L}\left(\mathscr{E}^{\prime}, \mathscr{F}^{\prime}\right)$, and $(y \otimes v)^{*}=$ $v \otimes y$. An ordered set $\left\{y_{0}, y_{1}, \ldots, y_{h}\right\} \subset \mathscr{E}$ is called a chain of eigenvectors and associated vectors $(\mathrm{CEAV})$ of $\mathscr{A}$ at $\lambda$ if

$$
Y=: \sum_{l=0}^{h}(\cdot-\lambda)^{l} y_{l}
$$

is a root function of $\mathscr{A}$ at $\lambda$ with $m(Y) \geq h+1$. Conversely, if $Y$ is a root function of $\mathscr{A}$ at $\lambda$ and $m(Y) \geq h+1$, then the function

$$
Y(\mu)=: \sum_{l=0}^{\infty}(\mu-\lambda)^{l} y_{l},
$$

$\left\{y_{0}, y_{1}, \ldots, y_{h}\right\}$ is CEAV. 
A system $\left\{y_{l}^{(j)}: 1 \leq j \leq h, 0 \leq l \leq m_{j}\right\}$ is called a canonical system of eigenvectors and associated vectors (CSEAV) of $\mathscr{A}$ at $\lambda$ if $\left\{y_{0}^{(j)}: 1 \leq j \leq h\right\}$ is a basis of $\operatorname{Ker}(\mathscr{A}(\lambda))$, $\left\{y_{0}^{(j)}, y_{1}^{(j)}, \ldots, y_{m j}^{(j)}\right\}$ is a CEAV of $\mathscr{A}$ at $\lambda(j=1, \ldots, h), m j=\sup \{m(y): y \in \operatorname{Ker}(\mathscr{A}(\lambda)) \backslash$ $\left.\operatorname{Span}\left\{y_{0}^{(k)}: k<j\right\}\right\} ;(1 \leq j \leq h)$. Obviously $m j=m\left(y_{0}^{(j)}\right)$.

We recall the generalization of Steinberg's theorem [15].

THEOREM 4.1. Suppose that $\mathscr{A}$ is an operator-valued function which is finitely meromorphic and of Fredholm type in the domain $D_{r_{0}}\left(\lambda_{0}\right)$. If the operator $\mathscr{A}(\lambda)$ is invertible at one point of $D_{r_{0}}$, then $\mathscr{A}(\lambda)$ has a bounded inverse for all $\lambda \in D_{r_{0}}$, except possibly for certain isolated points.

We will reduce, as mentioned in the introduction, the eigenvalue problem to some characteristic problem. From Proposition 3.1, we know that if $\lambda_{0}^{2}$ is an eigenvalue of (2.4), then $\lambda_{0}$ is a characteristic value of $L_{0}(\lambda)$. Moreover, for $r_{0}$ small enough, the function $L_{0}^{-1}(\lambda)$ is meromorphic in $\overline{D_{r_{0}}}\left(\lambda_{0}\right)$, where $D_{r_{0}}\left(\lambda_{0}\right)$ the disc of center $\lambda_{0}$ and radius $r_{0}$, and $\lambda_{0}$ is its unique pole in $\overline{D_{r_{0}}}$.

We begin by establishing the following lemma which characterises the eigenvalues of (2.4) if $\epsilon=0$.

Lemma 4.2. Any eigenvalue of the problem (2.4) is a simple pole of $L_{0}^{-1}(\lambda)$.

Proof. Let $\lambda_{0}^{2}$ be an eigenvalue of (2.4). Let $u_{0}$ be an associated eigenfunction normalized in $L^{2}\left(\Omega_{0}\right)$. Using an integration by parts, we know that $\partial_{\nu_{0}} u_{0}$ is in fact a characteristic function of the operator-valued function $\lambda \mapsto L_{0}(\lambda)$ corresponding to the characteristic value $\lambda_{0}$, that is, $L_{0}\left(\lambda_{0}\right) \partial_{\nu_{0}} u_{0}=0$ on $\partial \Omega_{0}$. We define $\phi(\lambda)$ as a root function of $L_{0}(\lambda)$ corresponding to $\left(\lambda_{0} ; \partial_{\nu_{0}} u_{0}\right)$; it is holomorphic in $D_{r_{0}}\left(\lambda_{0}\right), \phi\left(\lambda_{0}\right)=\partial_{\nu_{0}} u_{0}$ and satisfies the identity $\left.L_{0}(\lambda) \phi(\lambda)\right|_{\lambda=\lambda_{0}}=0$. The multiplicity of $\phi(\lambda)$ is the order of $\lambda_{0}$ as a zero of $L_{0}(\lambda) \phi(\lambda)$. But it is well known that the order of $\lambda_{0}$ as a pole of $L_{0}^{-1}(\lambda)$ is precisely the maximum of the ranks of the eigenvectors in $\operatorname{Ker} L_{0}\left(\lambda_{0}\right)$. Then it suffices to show that the rank of an arbitrary eigenvector is equal to one.

We write $L_{0}(\lambda) \phi(\lambda)=\left(\lambda^{2}-\lambda_{0}^{2}\right) \psi(\lambda)$, where $\psi(\lambda)$ is a holomorphic function in $H^{1 / 2}$ $\left(\partial \Omega_{0}\right)$. For $\lambda \in D_{r_{0}}\left(\lambda_{0}\right)$, we denote by $u(\lambda)$ the unique solution of $\left(\Delta+\lambda^{2}\right) u(\lambda)=0$ in $\Omega_{0}$ with the boundary condition $u(\lambda)=\psi(\lambda) \gamma^{-1}$ on $\partial \Omega_{0}$. Using a trivial integration by parts over $\Omega_{0}$, we find that

$$
\int_{\Omega_{0}} u(\lambda) \overline{u_{0}} d x=\left(\lambda^{2}-\lambda_{0}^{2}\right)^{-1} \int_{\partial \Omega_{0}} u(\lambda) \overline{\partial_{\nu_{0}} u_{0}} d \sigma(x)=\int_{\partial \Omega_{0}} \psi(\lambda) \overline{\partial_{\nu_{0}} u_{0}} d \sigma(x)
$$

which immediately implies that

$$
\int_{\partial \Omega_{0}} \psi\left(\lambda_{0}\right) \overline{\partial_{\nu_{0}} u_{0}} d \sigma(x)=\int_{\Omega_{0}}\left|u_{0}\right|^{2} d x=1,
$$

since $\int_{\Omega_{0}} u(\lambda) \overline{u_{0}} d x$ is holomorphic in $D_{r_{0}}\left(\lambda_{0}\right)$. Therefore, $\left|\psi\left(\lambda_{0}\right)\right|_{L^{2}\left(\partial \Omega_{0}\right)}^{2} \neq 0$ and thus the function $\psi\left(\lambda_{0}\right)$ is nontrivial.

Our main results in this section are summarized in the following theorem. 
Theorem 4.3. There exists a positive constant $\epsilon_{1}=\epsilon_{1}\left(r_{0}, \epsilon_{0}\right)$ such that $\epsilon_{1} \leq \epsilon_{0}$ and for $|\epsilon|<\epsilon_{1}$, the operator-valued function $\lambda \mapsto L_{\epsilon}(\lambda)$ has exactly $m$ characteristic values $\left(\lambda_{i}(\epsilon)\right)_{i}$ (counted according to their multiplicity) in $\overline{D_{r_{0}}}\left(\lambda_{0}\right)$. These characteristic values build the $\lambda_{0^{-}}$ group associated to the perturbed eigenvalue problem (2.4), and are analytic with respect to $\epsilon$ in $]-\epsilon_{1}, \epsilon_{1}\left[\right.$. They satisfy $\lambda_{i}(0)=\lambda_{0}$, for $i=1, \ldots, m$. Moreover, if $\left(\hat{\lambda}_{i}(\epsilon)\right)_{i=1}^{\tau_{\epsilon}}$ denotes the set of distinct values of $\left(\lambda_{i}(\epsilon)\right)_{i=1}^{m}$, then the following assertions hold:

$$
\begin{gathered}
\sum_{i=1}^{\tau_{\epsilon}} M\left(L_{\epsilon}\left(\hat{\lambda}_{i}(\epsilon)\right)\right)=m, \\
L_{\epsilon}^{-1}(\lambda)=\sum_{i=1}^{\tau_{\epsilon}}\left(\lambda-\hat{\lambda}_{i}(\epsilon)\right)^{-1} \ell_{i}(\epsilon)+\mathscr{R}_{\epsilon}(\lambda),
\end{gathered}
$$

where $\ell_{i}(\epsilon): \operatorname{Ker}\left(L_{\epsilon}\left(\hat{\lambda}_{i}(\epsilon)\right)\right) \rightarrow \operatorname{Ker}\left(L_{\epsilon}\left(\hat{\lambda}_{i}(\epsilon)\right)\right)$ and $\mathscr{R}_{\epsilon}(\lambda)$ is a holomorphic function with respect to $(\epsilon, \lambda) \in]-\epsilon_{0}, \epsilon_{0}\left[\times D_{r_{0}}\left(\lambda_{0}\right)\right.$.

Proof. We first recall that $m$ is the geometric multiplicity of $\lambda_{0}^{2}$ as an eigenvalue of the eigenvalue problem (2.4). Proposition 3.2 implies that $L_{\epsilon}(\lambda)$ is an analytic operator-valued function with respect to $(\epsilon, \lambda) \in \mathbb{R} \times D_{r_{0}}\left(\lambda_{0}\right)$. Then there exists a constant $\epsilon_{1}\left(r_{0}, \epsilon_{0}\right)>$ 0 such that for any $\epsilon$ lying in $]-\epsilon_{1}, \epsilon_{1}[$, the following holds:

$$
\left|\left(L_{\epsilon}(\lambda)-L_{0}(\lambda)\right) L_{0}^{-1}(\lambda)\right|_{\mathscr{L}\left(H_{\sharp}^{1 / 2}, H_{\sharp}^{1 / 2}\right)}<1, \quad \forall \lambda \in \partial D_{r_{0}}\left(\lambda_{0}\right) .
$$

From the generalized Rouché's theorem and the results of Gohberg and Sigal [6], we deduce that $L_{\epsilon}(\lambda)$ is invertible on $\partial D_{r_{0}}$ and has $\tau_{\epsilon}$ characteristic values $\left(\hat{\lambda}_{i}(\epsilon)\right)_{i}$ in $D_{r_{0}}\left(\lambda_{0}\right)$ which are (obviously) the poles of the function $L_{\epsilon}^{-1}(\lambda)$ in the disc $D_{r_{0}}\left(\lambda_{0}\right)$. Thus, with the definitions introduced earlier, the following holds:

$$
\sum_{i=1}^{\tau_{\epsilon}} M\left(L_{\epsilon}\left(\hat{\lambda}_{i}(\epsilon)\right)\right)=M\left(L_{0}\left(\lambda_{0}\right)\right) .
$$

Using Theorem 2.1 and Proposition 3.2, it can now be easily seen that the set of these characteristic values build precisely the $\lambda_{0}$-group of eigenvalues introduced in the last section, that is, $\left(\hat{\lambda}_{i}(\epsilon)\right)_{i} \equiv\left(\lambda_{i}(\epsilon)\right)_{i}$. Hence, they are analytic in the variable $\epsilon$. Notice that in general we have $M\left(L_{0}\left(\lambda_{0}\right)\right) \geq \operatorname{dim} \operatorname{Ker} L_{0}\left(\lambda_{0}\right)=m$. But Lemma 4.2 implies $M\left(L_{0}\left(\lambda_{0}\right)\right)=$ $m$. Furthermore, we have the Laurent expansion

$$
L_{0}^{-1}(\lambda)=\left(\lambda-\lambda_{0}\right)^{-1} \ell_{0}+\mathscr{R}_{0}(\lambda)
$$

where $\ell_{0}=\sum_{i=1}^{\tau_{0}} \ell_{i}(0)$ and $\mathscr{R}_{0}(\lambda)$ is a holomorphic function. From the decomposition (4.14), we obtain

$$
\begin{array}{ll}
\ell_{0} L_{0}\left(\lambda_{0}\right)=0 & \text { in } \mathscr{L}\left(H_{\#}^{-1 / 2}(] 0,1[), H_{\#}^{-1 / 2}(] 0,1[)\right), \\
L_{0}\left(\lambda_{0}\right) \ell_{0}=0 & \text { in } \mathscr{L}\left(H_{\#}^{1 / 2}(] 0,1[), H_{\#}^{1 / 2}(] 0,1[)\right) .
\end{array}
$$


1210 The integral equation methods for eigenvalue problems

It follows that $\ell_{0}: \operatorname{Ker} L_{0}^{*}\left(\lambda_{0}\right) \rightarrow \operatorname{Ker} L_{0}\left(\lambda_{0}\right)$. But from the properties of the Green's function $G(x, y)$, we know that

$$
\operatorname{Ker} L_{0}^{*}\left(\lambda_{0}\right)=\operatorname{Ker} L_{0}\left(\lambda_{0}\right)
$$

Note that using similar arguments, we can prove that $\left(\lambda_{i}(\epsilon)\right)_{i}$ are also simple poles of $L_{\epsilon}^{-1}(\lambda)$ and $M\left(L_{\epsilon}\left(\lambda_{i}(\epsilon)\right)\right)=\operatorname{dim} \operatorname{Ker} L_{\epsilon}\left(\lambda_{i}(\epsilon)\right)$, for $i=1, \ldots, m$. Moreover, we have

$$
\begin{array}{r}
L_{\epsilon}^{-1}(\lambda)=\sum_{i=1}^{\tau_{\epsilon}}\left(\lambda-\hat{\lambda}_{i}(\epsilon)\right)^{-1} \ell_{i}(\epsilon)+\mathscr{R}_{\epsilon}(\lambda), \\
\ell_{i}(\epsilon): \operatorname{Ker}\left(L_{\epsilon}\left(\hat{\lambda}_{i}(\epsilon)\right)\right) \longrightarrow \operatorname{Ker}\left(L_{\epsilon}\left(\hat{\lambda}_{i}(\epsilon)\right)\right),
\end{array}
$$

where $\mathscr{R}_{\epsilon}(\lambda)$ is a holomorphic function which completes the proof of the theorem.

Let $\lambda_{0}$ be a characteristic value of $L_{0}(\lambda)$. From Keldys's theorem which is simplified in [10, page 462], there exist $\left\{\phi_{0}^{i}: 1 \leq i \leq m\right\}$ CSEAV of $L_{0}$ at $\lambda_{0}$ and $\left\{\psi_{0}^{i}: 1 \leq i \leq m\right\}$ CSEAV of $L_{0}^{*}$ such that the operator

$$
A_{0}=\frac{1}{2 i \pi} \int_{\left|\lambda-\lambda_{0}\right|=\rho}\left(L_{0}(\lambda)\right)^{-1} d \lambda=\sum_{i=1}^{m} \phi_{0}^{i} \otimes \psi_{0}^{i}
$$

is well defined.

Analogously, by the result of Reinhard and Möller which is due to Keldyš [8], for each characteristic value $\lambda_{i}(\epsilon)(1 \leq i \leq m)$, there exist $\left\{\phi_{i, j}(\epsilon): 1 \leq i \leq m, 1 \leq j \leq m_{i}\right\}$ CSEAV of $L_{\epsilon}$ at $\lambda_{i}(\epsilon)$ and $\left\{\psi_{i, j}(\epsilon): 1 \leq i \leq m, 1 \leq j \leq m_{i}\right\}$ CSEAV of $L_{\epsilon}^{*}$ such that the operator

$$
A_{i}(\epsilon)=\frac{1}{2 i \pi} \int_{\left|\lambda-\lambda_{i}(\epsilon)\right|=\rho}\left(L_{\epsilon}(\lambda)\right)^{-1} d \lambda=\sum_{j=1}^{m_{i}} \phi_{i, j}(\epsilon) \otimes \psi_{i, j}(\epsilon)
$$

is well defined. Introduce the operator

$$
A(\epsilon)=\sum_{i=1}^{m} A_{i}(\epsilon), \quad \text { for }|\epsilon|<\epsilon_{1} .
$$

Based on Theorem 4.3, [15] and on relation (4.20), one can see that the operator $A(\epsilon)$ is selfadjoint and holomorphic function with respect to $\epsilon \in]-\epsilon_{1}, \epsilon_{1}$ [. It is quite easy to see that $A_{0}=A(\epsilon=0)$ and the following results hold.

Proposition 4.4. For $|\epsilon|<\epsilon_{1}$ and for $i \in\left\{1, \ldots, \tau_{\epsilon}\right\}$,

(1) the operator $A_{i}(\epsilon)$ satisfies

$$
\left.A_{i}(\epsilon)\right|_{\left(\operatorname{Ker}\left(L_{\epsilon}\left(\lambda_{i}(\epsilon)\right)\right)\right)^{\perp}} \equiv 0 ;
$$

(2) if $\lambda_{i}(\epsilon)$ and $\lambda_{j}(\epsilon)$ are two characteristic values of $L_{\epsilon}(\lambda)$ with $i \neq j$, then

$$
\operatorname{Ker} L_{\epsilon}\left(\lambda_{i}(\epsilon)\right) \perp \operatorname{Ker} L_{\epsilon}\left(\lambda_{j}(\epsilon)\right)
$$


Proof. (1) Let $\phi \in H_{\#}^{-1 / 2}$ such that $A_{i}(\epsilon) \phi=0$, then

$$
\sum_{j=1}^{m_{i}}\left(\phi, \psi_{i, j}(\epsilon)\right) \phi_{i, j}(\epsilon)=0
$$

The fact that $\left(\phi_{i j}\right)_{i j}$ and $\left(\psi_{i j}\right)_{i j}$ are CSEAV implies that $\phi=0$ and, therefore, yields the desired result.

(2) It suffices to prove the result only for $i=1$ and $j=2$. From relation (4.16), we deduce that

$$
L_{\epsilon}\left(\lambda_{1}(\epsilon)\right) \operatorname{Ker} L_{\epsilon}\left(\lambda_{2}(\epsilon)\right) \subset \operatorname{Ker} L_{\epsilon}\left(\lambda_{2}(\epsilon)\right)
$$

Then,

$$
L_{\epsilon}\left(\lambda_{1}(\epsilon)\right) \operatorname{Ker}\left(L_{\epsilon}\left(\lambda_{2}(\epsilon)\right)\right) \subset \operatorname{Ker}\left(L_{\epsilon}\left(\lambda_{2}(\epsilon)\right)\right) \cap\left(\operatorname{Ker}\left(L_{\epsilon}\left(\lambda_{1}(\epsilon)\right)\right)\right)^{\perp} .
$$

Using the last relation, the relation

$$
\operatorname{Ker}\left(L_{\epsilon}\left(\lambda_{2}(\epsilon)\right)\right)=L_{\epsilon}\left(\lambda_{1}(\epsilon)\right) \operatorname{Ker}\left(L_{\epsilon}\left(\lambda_{2}(\epsilon)\right)\right)+\left(I-L_{\epsilon}\left(\lambda_{1}(\epsilon)\right)\right) \operatorname{Ker}\left(L_{\epsilon}\left(\lambda_{2}(\epsilon)\right)\right)
$$

becomes

$$
\left(I-L_{\epsilon}\left(\lambda_{1}(\epsilon)\right)\right) \operatorname{Ker}\left(L_{\epsilon}\left(\lambda_{2}(\epsilon)\right)\right)=0 \text {. }
$$

The operator-valued function $L_{\epsilon}\left(\lambda_{1}(\epsilon)\right)$ is Fredholm of index 0 which completes the proof.

Our strategy now is to investigate the properties of the eigenelements corresponding to the operators $A_{0}$ and $A_{\epsilon}$. Let $\left(\mu_{0}^{j}\right)_{1 \leq j \leq h}$ be the family of eigenvalues of the operator $A_{0}$ with multiplicity $m_{j}$ each. Using the generalisation of Theorem 2.1 , see $[7,13]$, we know that there exists $\epsilon_{2}=\epsilon_{2}\left(\epsilon_{1}\right)>0$ such that for $|\epsilon|<\epsilon_{2}$ and for $j \in\{1, \ldots, h\}$, the $\mu_{0}^{j}$ group consists of $m_{j}$ eigenvalues of $A(\epsilon), \mu_{j, l}(\epsilon), l=1, \ldots, m_{j}$ (repeated according to their multiplicity).

Let $\epsilon_{3}=\inf \left(\epsilon_{1}, \epsilon_{2}\right)$. For $|\epsilon|<\epsilon_{3}$, the following projector is well defined:

$$
P_{j}(\epsilon)=\frac{1}{2 i \pi} \int_{\left|\mu-\mu_{j, i}(\epsilon)\right|=\rho_{1}}(\mu-A(\epsilon))^{-1} d \mu=\sum_{l=1}^{m_{j}} \sum_{s=1}^{m_{j, l}} q_{l, s}^{(j)}(\epsilon) \otimes q_{l, s}^{(j)}(\epsilon),
$$

where for $1 \leq j \leq h$ and for $1 \leq l \leq m_{j}$, the family $\left(q_{l, s}^{(j)}(\epsilon)\right)_{1 \leq s \leq m_{j l}}$ denotes the orthogonal family of eigenfunctions corresponding to the eigenvalues $\mu_{j, l}(\epsilon)$. For $\epsilon=0$, we have

$$
P_{j}(0)=\frac{1}{2 i \pi} \int_{\left|\mu-\mu_{0}^{j}\right|=\rho_{2}}(\mu-A(0))^{-1} d \mu=\sum_{l=1}^{m_{j}} q_{l}^{(j)}(0) \otimes q_{l}^{(j)}(0),
$$




\section{The integral equation methods for eigenvalue problems}

where the family $\left(q_{l}^{(j)}(0)\right)_{1 \leq l \leq m_{j}}$ is the orthogonal family of eigenfunctions corresponding to the eigenvalue $\mu_{0}^{j}$. Now it seems natural, from the previous results, that for all $j=1, \ldots, h$, the family $\left(q_{l}^{(j)}(0)\right)_{1 \leq l \leq m_{j}}$ is $m_{j}$-characteristic functions of $L_{0}\left(\lambda_{0}\right)$ and for all $l=1, \ldots, m_{j}$, the family $\left(q_{l, s}^{(j)}(\epsilon)\right)_{1 \leq s \leq m_{j, l}}$ is $m_{j, l}$-characteristic functions of $L_{\epsilon}\left(\lambda_{i}(\epsilon)\right)$ and $\sum_{j=1}^{h} m_{j}=m$.

We set

$$
P(\epsilon)=\sum_{j=1}^{h} P_{j}(\epsilon), \quad \text { for }|\epsilon|<\epsilon_{3}, \quad P(0)=\sum_{j=1}^{h} P_{j}(0) .
$$

\section{Analyticity and asymptotic expansion}

This section is devoted to the study of the asymptotics of the characteristic elements and, therefore, the asymptotics of the eigenelements of (2.4) when the parameter $\epsilon$ goes to zero. We will give a method in order to calculate the coefficients of the expansions of the eigenelements in a neighborhood of zero when the eigenvalue $\lambda_{0}^{2}$ of $-\Delta$ is not simple. Our strategy, for deriving asymptotic expansions of the perturbations in a multiple eigenvalue $\lambda_{0}$ with multiplicity $m$ that are due to boundary deformations, relies on finding the analyticity and complete asymptotic expansions of the eigenelements of $A(\epsilon)$. The following holds.

Proposition 5.1. For $|\epsilon|<\epsilon_{3}$,

(1) the operator $P(\epsilon)$ is holomorphic for $\epsilon \in]-\epsilon_{3}, \epsilon_{3}[$ and $P(\epsilon)=P(0)+R(\epsilon)$, where $R(\epsilon)$ is holomorphic with respect to $\epsilon$,

(2) $P(\epsilon)=\sum_{j=1}^{m} q^{(j)}(\epsilon) \otimes q^{(j)}(\epsilon)$, where $\left(q^{(j)}(\epsilon)\right)_{1 \leq j \leq m}$ denotes an orthonormal basis of $\operatorname{Ker} L_{\epsilon}\left(\lambda_{i}(\epsilon)\right)$. Also, $P(0)=\sum_{j=1}^{m} q^{(j)}(0) \otimes q^{(j)}(0)$, where $\left(q^{(j)}(0)\right)_{1 \leq j \leq m}$ is an orthonormal basis of $\operatorname{Ker} L_{0}\left(\lambda_{0}\right)$.

Proof. (1) This property is clear by recalling that the operator $A(\epsilon)$ is holomorphic and has the expansion $A(\epsilon)=A(0)+\widetilde{A}(\epsilon)$, where the operator $\widetilde{A}(\epsilon)$ is holomorphic with respect to $\epsilon$ and goes to 0 as $\epsilon \rightarrow 0$ and by considering, for $\epsilon \in]-\epsilon_{3}, \epsilon_{3}$ [, the Neumann series

$$
(\mu-A(\epsilon))^{-1}=(\mu-A(0))^{-1}+\sum_{p=1}^{\infty}(\mu-A(0))^{-1}\left[\widetilde{A}(\epsilon)(\mu-A(0))^{-1}\right]^{p},
$$

which converges uniformly with respect to $\mu$ in a neighborhood of $\mu_{j, i}$.

(2) As said at the end of Section 4, the elements of the family $\left(q_{l}^{(j)}(0)\right)_{1 \leq j \leq h, 1 \leq l \leq m_{j}}$ are characteristic functions of $L_{0}\left(\lambda_{0}\right)$ and the idea here is to organize this family as follows:

$$
q_{1}^{(1)}(0)=q_{0}^{(1)}, q_{2}^{(1)}(0)=q_{0}^{(2)}, \ldots, q_{m_{h}}^{(h)}(0)=q_{0}^{(m)} .
$$

Then, the family $\left(q_{0}^{(j)}\right)_{1 \leq j \leq m}$ defines an orthogonal basis in $\overline{\operatorname{Ker} L_{0}\left(\lambda_{0}\right)}$. Using similar arguments, the family $\left(q_{l, s}^{(j)}(\epsilon)\right)_{1 \leq l \leq m_{j}, 1 \leq s \leq m_{j l}, 1 \leq j \leq h}$, given by (4.28), can be organized:

$$
q_{1,1}^{(1)}(\epsilon)=q^{(1)}(\epsilon), q_{1,2}^{(1)}(0)=q^{(2)}(\epsilon), \ldots, q_{m_{h}, m_{h m_{h}}}^{(h)}(\epsilon)=q^{(m)}(\epsilon)
$$


where for all $j=1, \ldots, m, q^{(j)}(\epsilon) \rightarrow q_{0}^{(j)}$ as $\epsilon \rightarrow 0$. The family $\left(q^{(j)}(\epsilon)\right)_{1 \leq j \leq m}$ defines an orthogonal basis in $\bigoplus_{i=1}^{m} \operatorname{Ker}\left(L_{\epsilon}\left(\lambda_{i}(\epsilon)\right)\right)$, and so the order of organization of its elements directly depends on the order of organization of the basis $\left(q_{0}^{(j)}\right)_{1 \leq j \leq m}$.

Let $B_{\epsilon}=\left[a_{l s}\right]$ be the $(m \times m)$ matrix, where for $l=1, \ldots, m$ and for $s=1, \ldots, m$,

$$
a_{l s}=\left(q_{0}^{(l)}, q_{\epsilon}^{(s)}\right)
$$

Then, $B_{0}=I_{m}$, where $I_{m}$ denotes the identity matrix. From Proposition 5.1, we have

$$
\sum_{j=1}^{m}\left(\cdot, q^{(j)}(\epsilon)\right) q^{(j)}(\epsilon)=\sum_{j=1}^{m}\left(\cdot, q_{0}^{(j)}\right) q_{0}^{(j)}+R(\epsilon) .
$$

Relation (5.5) implies that

$$
\left(B_{\epsilon} \phi_{\epsilon}\right)_{l}=q_{0}^{(l)}+R(\epsilon) q_{0}^{(l)}
$$

where

$$
q_{0}=\left(q_{0}^{(1)}, \ldots, q_{0}^{(m)}\right)^{T}, \quad \phi_{\epsilon}=\left(q^{(1)}(\epsilon), \ldots, q^{(m)}(\epsilon)\right)^{T} .
$$

Proposition 5.2. There exists some constant $\epsilon_{4}=\epsilon_{4}\left(\epsilon_{3}\right)>0,\left(\epsilon_{4} \leq \epsilon_{3}\right)$, such that for $j \in$ $\{1, \ldots, m\}$,

(1) the functions $q^{(j)}(\epsilon)(t)$ are holomorphic in $(t, \epsilon)$ and satisfy the following uniform expansions: for $t \in[0,1]$,

$$
q^{(j)}(\epsilon)(t)=q_{0}^{(j)}(t)+\sum_{n \geq 1} q_{n}^{(j)}(t) \epsilon^{n}
$$

where the first coefficient satisfies

$$
q_{1}^{(j)}=R_{1} q_{0}^{(j)}
$$

and for $n \geq 2$, the coefficients $q_{n}^{(j)}$ are given by

$$
q_{n}^{(j)}=R_{n} q_{0}^{(j)}-\sum_{k=1}^{n-1} \sum_{i=1}^{m}\left(q_{0}^{(j)}, q_{k}^{(i)}\right) q_{n-k}^{(i)},
$$

with $R_{0}=0$ and $R_{n}(n \geq 1)$ being the Taylor coefficients of $R(\epsilon)$,

(2) the characteristic values $\lambda_{j}(\epsilon)=\lambda^{(j)}(\epsilon)$ satisfy

$$
\lambda^{(j)}(\epsilon)=\lambda_{0}+\sum_{n \geq 1} \lambda_{n}^{(j)} \epsilon^{n} .
$$

The first coefficient satisfies

$$
\lambda_{1}^{(j)}=-1-\frac{\left(L_{0}\left(\lambda_{0}\right) q_{1}^{(j)}, l_{1}^{(0)} q_{0}^{(j)}\right)}{\left\|l_{1}^{(0)} q_{0}^{(j)}\right\|^{2}}
$$


1214 The integral equation methods for eigenvalue problems

and for $n \geq 2$,

$$
\begin{aligned}
\lambda_{n}^{(j)}=-\frac{1}{\left\|l_{1}^{(0)} q_{0}^{(j)}\right\|^{2}}[ & \sum_{i=2}^{n}\left(\sum_{s_{1}+\cdots+s_{i}=n} \lambda_{s_{1}}^{(j)} \lambda_{s_{2}}^{(j)} \cdots \lambda_{s_{i}}^{(j)}\right)\left(l_{i}^{(0)} q_{0}^{(j)}, l_{1}^{(0)} q_{0}^{(j)}\right) \\
& \left.+\sum_{l=0}^{n-1} \sum_{k=0}^{l}\left(F_{k, l-k} q_{n-l}^{(j)}, l_{1}^{(0)} q_{0}^{(j)}\right)+\sum_{k=1}^{n}\left(F_{k, n-k} q_{0}^{(j)}, l_{1}^{(0)} q_{0}^{(j)}\right)\right]
\end{aligned}
$$

Here for all integers $k$ and $s$, the expressions $l_{k}^{(s)}$ and $F_{k, s}$ are two operator-valued functions with simple forms.

Proof. (1) Define the matrix: $\mathscr{D}_{\epsilon}=\left(d_{s p}^{\epsilon}\right)_{s p}$; the coefficients $d_{s p}^{\epsilon}$ are given by

$$
d_{s p}^{\epsilon}=\delta_{s}^{p}+\left(R(\epsilon) q_{0}^{(s)}, q_{0}^{(p)}\right), \quad\left(\delta_{s}^{p}=1 \text { if } p=s \text { and } \delta_{s}^{p}=0 \text { if } p \neq s\right)
$$

The analyticity of the operator-valued function $R(\epsilon)$ with respect to $\epsilon \in]-\epsilon_{3}, \epsilon_{3}$ [ guarantees the analyticity of $\mathscr{D}_{\epsilon}$. The inner product of (5.5) by $q_{0}^{(p)}$ gives

$$
\sum_{l=1}^{m}\left(q_{0}^{(s)}, q^{(l)}(\epsilon)\right)\left(q^{(l)}(\epsilon), q_{0}^{(p)}\right)=\delta_{s}^{p}+\left(R(\epsilon) q_{0}^{(s)}, q_{0}^{(p)}\right)
$$

which implies that

$$
B_{\epsilon}^{2}=\mathscr{D}_{\epsilon}
$$

Relation (5.5) implies

$$
B_{\epsilon} \phi_{\epsilon}=\phi_{0}+R(\epsilon) \phi_{0}
$$

We now verify that the function $\phi_{\epsilon}$ is jointly analytic in $(t, \epsilon)$. Through relation (5.17), we deduce that

$$
\phi_{\epsilon}=\left(B_{\epsilon}\right)^{-1}\left(\phi_{0}+R(\epsilon) \phi_{0}\right)
$$

The analyticity of $\phi_{0}(t)$ in $t$ is a classical result. Then we deduce the result by using the analyticity of the matrix $B_{\epsilon}^{-1}$ in $\epsilon$, which is obvious from (5.16), and the fact that the function $R(\epsilon) \phi_{0}(t)$ is jointly analytic in $(t, \epsilon)$. From relation (5.7), we then deduce the analyticity of the functions $q^{(j)}(\epsilon)$ follows for all $j=1, \ldots, m$.

The analyticity of the matrix operator $B_{\epsilon}$ allows writing, in a neighborhood of 0 , the expansion

$$
B_{\epsilon}=B_{0}+\epsilon B_{1}+\epsilon^{2} B_{2}+\cdots
$$

By expanding

$$
\phi_{\epsilon}=\sum_{k \geq 0} \epsilon^{k} q_{k}, \quad \text { for }|\epsilon|<\epsilon_{4},
$$


and (5.7), relation (5.17) implies

$$
\left(\sum_{n=0}^{+\infty} \epsilon^{n} B_{n}\right)\left(\sum_{s=0}^{+\infty} \epsilon^{s} q_{s}\right)=q_{0}+\sum_{n=1}^{+\infty} \epsilon^{n} R_{n} q_{0}
$$

where we have considered $\phi_{0}=q_{0}$ and $R(\epsilon)=\sum_{n \geq 1} \epsilon^{n} R_{n}$. Then,

$$
\sum_{k=0}^{n} B_{k} q_{n-k}=q_{0}+R_{n} q_{0}
$$

The $j$ th component of the vector $B_{k} q_{n-k}$ is given by

$$
\left(B_{k} q_{n-k}\right)_{j}=\sum_{i=1}^{m}\left(q_{0}^{(j)}, q_{k}^{(i)}\right) q_{n-k}^{(i)}
$$

and then relation (5.22) becomes

$$
\left(B_{0} q_{n}\right)_{j}+\sum_{k=1}^{n} \sum_{i=1}^{m}\left(q_{0}^{(j)}, q_{k}^{(i)}\right) q_{n-k}^{(i)}=q_{0}^{(j)}+R_{n} q_{0}^{(j)}
$$

The relation $B_{0}=I_{m}$ implies

$$
q_{n}^{(j)}=-\sum_{k=1}^{n} \sum_{i=1}^{m}\left(q_{0}^{(j)}, q_{k}^{(i)}\right) q_{n-k}^{(i)}+q_{0}^{(j)}+R_{n} q_{0}^{(j)} .
$$

Therefore,

$$
q_{n}^{(j)}=-\sum_{i=1}^{m}\left(q_{0}^{(j)}, q_{n}^{(i)}\right) q_{0}^{(i)}-\sum_{k=1}^{n-1} \sum_{i=1}^{m}\left(q_{0}^{(j)}, q_{k}^{(i)}\right) q_{n-k}^{(i)}+q_{0}^{(j)}+R_{n} q_{0}^{(j)}
$$

Taking the inner product with $q_{0}^{(s)}, s=1, \ldots, m$, we have

$$
\left(q_{0}^{(s)}, q_{n}^{(j)}\right)=-\left(q_{0}^{(j)}, q_{n}^{(s)}\right)-\sum_{k=1}^{n-1} \sum_{i=1}^{m}\left(q_{0}^{(j)}, q_{k}^{(i)}\right)\left(q_{0}^{(s)}, q_{n-k}^{(i)}\right)+\delta_{s j}+\left(q_{0}^{(s)}, R_{n} q_{0}^{(j)}\right)
$$

Then,

$$
\left(q_{0}^{(j)}, q_{n}^{(s)}\right)=-\left(q_{0}^{(s)}, q_{n}^{(j)}\right)-\sum_{k=1}^{n-1} \sum_{i=1}^{m}\left(q_{0}^{(j)}, q_{k}^{(i)}\right)\left(q_{0}^{(s)}, q_{n-k}^{(i)}\right)+\delta_{s j}+\left(q_{0}^{(s)}, R_{n} q_{0}^{(j)}\right) .
$$

If we replace this equality in (5.26), we find

$$
\begin{aligned}
q_{n}^{(j)}= & \sum_{i=1}^{m}\left(q_{0}^{(i)}, q_{n}^{(j)}\right) q_{0}^{(i)}+\sum_{s=1}^{m}\left[\sum_{k=1}^{n-1} \sum_{i=1}^{m}\left(q_{0}^{(j)}, q_{k}^{(i)}\right)\left(q_{0}^{(s)}, q_{n-k}^{(i)}\right)-\delta_{s j}-\left(q_{0}^{(s)}, R_{n} q_{0}^{(j)}\right)\right] q_{0}^{(s)} \\
& -\sum_{k=1}^{n-1} \sum_{i=1}^{m}\left(q_{0}^{(j)}, q_{k}^{(i)}\right) q_{n-k}^{(i)}+q_{0}^{(j)}+R_{n} q_{0}^{(j)} .
\end{aligned}
$$


1216 The integral equation methods for eigenvalue problems

We recall that

$$
P(0) q_{n}^{(j)}=\sum_{i=1}^{m}\left(q_{0}^{(i)}, q_{n}^{(j)}\right) q_{0}^{(i)}
$$

Then relation (5.29) becomes

$$
\begin{aligned}
\left(I-P_{0}\right) q_{n}^{(j)}= & \sum_{s=1}^{m}\left[\sum_{k=1}^{n-1} \sum_{i=1}^{m}\left(q_{0}^{(j)}, q_{k}^{(i)}\right)\left(q_{0}^{(s)}, q_{n-k}^{(i)}\right)-\delta_{s j}-\left(q_{0}^{(s)}, R_{n} q_{0}^{(j)}\right)\right] q_{0}^{(s)} \\
& -\sum_{k=1}^{n-1} \sum_{i=1}^{m}\left(q_{0}^{(j)}, q_{k}^{(i)}\right) q_{n-k}^{(i)}+q_{0}^{(j)}+R_{n} q_{0}^{(j)} .
\end{aligned}
$$

From the previous relation and the properties of the operator $I-P_{0}$, we deduce

$$
\left(I-P_{0}\right)\left(q_{n}^{(j)}+\sum_{k=1}^{n-1} \sum_{i=1}^{m}\left(q_{0}^{(j)}, q_{k}^{(i)}\right) q_{n-k}^{(i)}-R_{n} q_{0}^{(j)}\right)=0
$$

In other words, it is obvious that $R_{n} q_{0}^{(j)} \notin \operatorname{Ker}\left(L_{0}\left(\lambda_{0}\right)\right)$ for all $j=1, \ldots, m$. Then

$$
\left(q_{n}^{(j)}+\sum_{k=1}^{n-1} \sum_{i=1}^{m}\left(q_{0}^{(j)}, q_{k}^{(i)}\right) q_{n-k}^{(i)}-R_{n} q_{0}^{(j)}\right) \notin \operatorname{Ker}\left(L_{0}\left(\lambda_{0}\right)\right)
$$

Thus, relation (5.32) means that

$$
q_{n}^{(j)}+\sum_{k=1}^{n-1} \sum_{i=1}^{m}\left(q_{0}^{(j)}, q_{k}^{(i)}\right) q_{n-k}^{(i)}-R_{n} q_{0}^{(j)}=0 .
$$

(2) In order to find out the coefficients in (5.11), our method is based on expanding the expression $L_{\epsilon}\left(\lambda^{(j)}(\epsilon)\right)$ for $\epsilon$ near zero. To handle this, we have to expand, first, the operator-valued function $L_{\epsilon}(\lambda)$ around $\epsilon=0$ and so the resulting expression around $\lambda=$ $\lambda_{0}$.

We first recall the formula (see [1] and [3, page 332])

$$
H_{0}^{(1)}(z)=J_{0}(z)+i Y_{0}(z)
$$

where $J_{0}$ and $Y_{0}$ are the Bessel functions

$$
\begin{gathered}
J_{0}(z)=\sum_{n=0}^{+\infty} \frac{(-1)^{n}}{(n !)^{2}}\left(\frac{z}{2}\right)^{2 n}, \\
Y_{0}(z)=\frac{2}{\pi}\left(\Gamma_{0}+\log \left(\frac{z}{2}\right)\right) J_{0}(z)-\frac{2}{\pi} \sum_{n=1}^{+\infty} \frac{(-1)^{n}}{(n !)^{2}}\left(\frac{z}{2}\right)^{2 n}\left(1+\frac{1}{2}+\cdots \frac{1}{n}\right) .
\end{gathered}
$$


Thus, in particular, we can write

$$
H_{0}^{(1)}(z)=h_{1}\left(z^{2}\right)+h_{2}\left(z^{2}\right) \log (z)=h_{1}\left(z^{2}\right)+\left(\Gamma_{0}+z^{2} h_{3}\left(z^{2}\right)\right) \log (z)
$$

for some entire functions $h_{1}, h_{2}$, and $h_{3}$, with $\Gamma_{0}$ being a real constant.

Combining the last result and the kernel of the operator $L_{\epsilon}(\lambda)$ (see Proposition 3.1), we formally obtain the following uniform expansion: for all $(\lambda, t, s) \in D_{r_{0}}\left(\lambda_{0}\right) \times[0,1] \times[0,1]$,

$$
G(\lambda, t, s)=\frac{i}{4} H_{0}^{(1)}\left(\lambda\left|\gamma_{\epsilon}(t)-\gamma_{\epsilon}(s)\right|\right)=\sum_{n \geq 0} G_{n}(\lambda, t, s) \epsilon^{n}
$$

with the Taylor coefficients

$$
\begin{aligned}
G_{n}(\lambda, t, s)=\frac{i}{4 n !} \sum_{k=0}^{n} & \lambda^{k}\left(\frac{(\beta(t)-\beta(s), \gamma(t)-\gamma(s))}{|\gamma(t)-\gamma(s)|}\right)^{k}\left(\frac{\left(\beta^{\prime}(s), \gamma^{\prime}(s)\right)}{\left|\gamma^{\prime}(s)\right|}\right)^{n-k} \\
& \times \frac{d^{k} H_{0}^{(1)}}{d z^{k}}(\lambda|\gamma(t)-\gamma(s)|) .
\end{aligned}
$$

As an immediate consequence, the following holds:

$$
L_{\epsilon}(\lambda)=\sum_{n=0}^{+\infty} L_{n}(\lambda) \epsilon^{n}, \quad \text { for } \epsilon \text { small enough. }
$$

By considering Proposition 3.1, it seems clear that each operator $L_{n}(\lambda)$ has kernel $G_{n}(\lambda$, $t, s)$. One also gets from (5.39) the following uniform expansion: for $(t, s) \in[0,1] \times[0,1]$,

$$
G_{n}(\lambda, t, s)=\sum_{k=0}^{+\infty}\left(\lambda-\lambda_{0}\right)^{k} G_{k}^{(n)}(t, s)
$$

where $\lambda$ is in a neighborhood of $\lambda_{0}$, with the coefficients

$$
\begin{aligned}
G_{k}^{(n)}(t, s)=\frac{i}{4 n !} \sum_{i=0}^{n} \sum_{l=0}^{k} & \frac{1}{l !(k-l) !} \frac{[(\beta(t)-\beta(s), \gamma(t)-\gamma(s))]^{i}}{|\gamma(t)-\gamma(s)|^{i+l-k}} \\
& \times\left.\left(\frac{\left(\beta^{\prime}(s), \gamma^{\prime}(s)\right)}{\left|\gamma^{\prime}(s)\right|}\right)^{n-i} \frac{d^{l}\left(\lambda^{i}\right)}{d \lambda^{l}}\right|_{\lambda=\lambda_{0}} \frac{d^{k+i-l}}{d z^{k+i-l}} H_{0}^{(1)}\left(\lambda_{0}|\gamma(t)-\gamma(s)|\right) .
\end{aligned}
$$

Now, for $(\epsilon, \lambda)$ in a neighborhood of $\left(0, \lambda_{0}\right)$, we have the following expansions:

$$
L_{\epsilon}(\lambda)=\sum_{n=0}^{+\infty} \sum_{k=0}^{+\infty} \epsilon^{n}\left(\lambda-\lambda_{0}\right)^{k} l_{k}^{(n)}
$$


1218 The integral equation methods for eigenvalue problems

where the operator $l_{k}^{(n)}$ has kernel $G_{k}^{(n)}(t, s)$. We know that $\lambda^{(j)}(\epsilon) \rightarrow \lambda_{0}$ as $\epsilon \rightarrow 0$. Then for $\epsilon$ small enough, we can replace $\lambda^{(j)}(\epsilon)$ given by (5.11) in relation (5.43), and so we obtain

$$
L_{\epsilon}\left(\lambda^{(j)}(\epsilon)\right)=\sum_{n=0}^{+\infty}\left(\sum_{k=0}^{n} F_{k, n-k}\right) \epsilon^{n}
$$

where

$$
\begin{gathered}
F_{n, 0}=l_{0}^{(n)} \\
F_{n, k}=\sum_{i=1}^{k}\left(\sum_{s_{1}+\cdots+s_{i}=k} \lambda_{s_{1}}^{(j)} \lambda_{s_{2}}^{(j)} \cdots \lambda_{s_{i}}^{(j)}\right) l_{i}^{(n)} .
\end{gathered}
$$

Remember that

$$
L_{\epsilon}\left(\lambda^{(j)}(\epsilon)\right) q^{(j)}(\epsilon)=0, \quad \forall j=1, \ldots, m .
$$

Then by using (5.10) and (5.44) at order $n \geq 1$, we can easily write

$$
\sum_{l=0}^{n} \sum_{k=0}^{l} F_{k, l-k} q_{n-l}^{(j)}=0 .
$$

Thus, from (5.45) and by simple calculus, we find for $n \geq 2$,

$$
\begin{aligned}
\lambda_{n}^{(j)}=-\frac{1}{\left\|l_{1}^{(0)} q_{0}^{(j)}\right\|^{2}}[ & \sum_{i=2}^{n}\left(\sum_{s_{1}+\cdots+s_{i}=n} \lambda_{s_{1}}^{(j)} \lambda_{s_{2}}^{(j)} \cdots \lambda_{s_{i}}^{(j)}\right)\left(l_{i}^{(0)} q_{0}^{(j)}, l_{1}^{(0)} q_{0}^{(j)}\right) \\
& \left.+\sum_{l=0}^{n-1} \sum_{k=0}^{l}\left(F_{k, l-k} q_{n-l}^{(j)}, l_{1}^{(0)} q_{0}^{(j)}\right)+\sum_{k=1}^{n}\left(F_{k, n-k} q_{0}^{(j)}, l_{1}^{(0)} q_{0}^{(j)}\right)\right] .
\end{aligned}
$$

We now give the following lemma which seems useful to prove the fundamental result in this section.

Lemma 5.3. Let $\mathcal{K}_{0}$ be a bounded neighborhood of $\overline{\Omega_{0}}$ in $\mathbb{R}^{2}$. The functions $\hat{u}_{i, j}(\epsilon)(x)=$ $S\left(\hat{\lambda}_{j}(\epsilon)\right) q^{(i)}(\epsilon)\left(\gamma^{-1}\right)$ are jointly analytic in the variables $\left.(x, \epsilon) \in \mathcal{K}_{0} \times\right]-\epsilon_{4}, \epsilon_{4}[$.

Proof. The function $\hat{u}_{i, j}(\epsilon)(x)=S\left(\hat{\lambda}_{j}(\epsilon)\right) q^{(i)}(\epsilon)\left(\gamma^{-1}\right)$ satisfies the Helmholtz equation in $\Omega_{\epsilon}$ with the boundary conditions $\hat{u}_{i, j}(\epsilon) \mid \partial \Omega_{\epsilon}=0$ and $\partial_{v_{\epsilon}} \hat{u}_{i, j}(\epsilon)\left(\gamma_{\epsilon}(t)\right)=q^{(j)}(\epsilon)(t)$, which are jointly analytic with respect to the variables $(t, \epsilon) \in[0,1] \times]-\epsilon_{4}, \epsilon_{4}[$. The outward unit normal $v_{\epsilon}$ to $\partial \Omega_{\epsilon}$ is given by $\gamma_{\epsilon}^{\prime}(t) /\left|\gamma_{\epsilon}^{\prime}(t)\right|$, as a function of $t=\gamma^{-1}(x)$. The symbol of the operator $\Delta_{x}=\partial_{x_{1}}^{2}+\partial_{x_{2}}^{2}$ is $\mathscr{S}\left(\xi_{1}, \xi_{2}, \epsilon\right)=\xi_{1}^{2}+\xi_{2}^{2}$. Thus $\mathscr{S}\left(\nu_{\epsilon}\right)=1>0$. Since the surface $\partial \Omega_{\epsilon}$ is noncharacteristic for $\Delta_{x}$, the Cauchy-Kowaleski theorem implies that $\hat{u}_{i, j}(\epsilon)(x)$ is jointly analytic with respect to $(x, \epsilon)$ in $\left.\left\{|x-\gamma(t)| \leq \alpha_{0}\right\} \times\right]-\epsilon_{4}, \epsilon_{4}[$, where $\alpha_{0}$ is a positive constant.

The following result holds. 
Theorem 5.4. Let $\mathscr{K}_{0}$ be a bounded neighborhood of $\bar{\Omega}_{0}$ in $\mathbb{R}^{2}$. Then there exists a constant $\epsilon_{5}>0$ smaller than $\epsilon_{4}$ such that an orthonormal basis of eigenfunctions $\left(u_{j}(\epsilon)\right)_{j}$ corresponding to the $\lambda_{0}$-group, $\left(\lambda_{j}(\epsilon)\right)_{j}$, in $H_{0}^{1}\left(\Omega_{\epsilon}\right)$ can be chosen to depend holomorphically on $\left.(x, \epsilon) \in \mathcal{K}_{0} \times\right]-\epsilon_{5}, \epsilon_{5}[$. Moreover, these eigenfunctions satisfy the following uniform expansion: for $x \in \mathcal{K}_{0}$,

$$
u_{j}(\epsilon)=u_{0}^{(j)}+\sum_{n \geq 1} u_{n}^{(j)} \epsilon^{n}
$$

where the family $u_{0}^{(j)}$ builds a basis of eigenfunctions of (2.4) associated to $\lambda_{0}^{2}$ and normalized in $L^{2}\left(\Omega_{0}\right)$. The terms $u_{n}^{(j)}$ are computed from the Taylor coefficients of $q^{(j)}(\epsilon)$.

Proof. Propositions 5.1 and 5.2 imply that there exists an orthonormal basis $\left(q^{(i)}(\epsilon)\right)_{1 \leq i \leq m j}(t, \epsilon) \in H_{\#}^{-1 / 2}(] 0,1[)$ of $\operatorname{Ker}\left(L_{\epsilon}\left(\hat{\lambda}^{(j)}(\epsilon)\right)\right)$, which is analytic in $(t, \epsilon) \in[0,1] \times$ ]$-\epsilon_{4}, \epsilon_{4}\left[\right.$. We know that $S\left(\hat{\lambda}_{j}(\epsilon)\right) q^{(i)}(\epsilon)\left(\gamma^{-1}\right)$ builds a basis of eigenfunctions of the eigenvalue problem (2.4) associated to $\hat{\lambda}_{j}^{2}(\epsilon)$. Using the Schmidt orthogonalization process once again, we construct the desired orthonormal basis. Clearly, the functions $\left(\hat{u}_{i, j}(\epsilon)\right)_{i j}$, introduced in Lemma 5.3, build a basis of the eigenspaces corresponding to the $\lambda_{0}$-group, $\left(\lambda_{j}(\epsilon)\right)_{j}$ in $H_{0}^{1}\left(\Omega_{\epsilon}\right)$. We will now give the asymptotic expansion of these functions when $\epsilon$ tends to 0 . To simplify notations, we drop the subscripts $i$ and $j$. Integral equations give

$$
\widehat{u}(\epsilon)(x)=\int_{0}^{1} G\left(\lambda(\epsilon)\left|x-\gamma_{\epsilon}(t)\right|\right) q(\epsilon)(t)\left|\gamma_{\epsilon}^{\prime}(t)\right| d t, \quad x \in \mathcal{K}_{0}
$$

The perturbed eigenvalue $\lambda(\epsilon)$ lies in a small neighborhood of $\lambda_{0}$ for small values of $\epsilon$. Then, there exists $\epsilon_{5}>0\left(\epsilon_{5} \leq \epsilon_{4}\right)$, such that we have the following Taylor expansion:

$$
G\left(\lambda(\epsilon)\left|x-\gamma_{\epsilon}(t)\right|\right)\left|\gamma_{\epsilon}^{\prime}(t)\right|=G\left(\lambda_{0}|x-\gamma(t)|\right)\left|\gamma^{\prime}(t)\right|+\sum_{k \geq 1} \epsilon^{k} G_{k}(x, t)
$$

which holds uniformly in $x \in \mathcal{K}_{0}$ and $t \in[0,1]$. We use Proposition 5.2 to write

$$
q(\epsilon)(t)=q_{0}(t)+\sum_{k \geq 1} \epsilon^{k} q_{k}(t)
$$

uniformly in $t \in[0,1]$. Substituting the last two expansions into (5.50), we find

$$
\widehat{u}(\epsilon)=\widehat{u}(0)+\sum_{k \geq 1} \epsilon^{k}\left[\sum_{n=1}^{k} \int_{0}^{1} q_{k-n}(t) G_{n}(x, t) d t\right] .
$$

Now we use the Schmidt orthogonalization process to construct from the eigenfunctions $\left(\hat{u}_{j}(\epsilon)\right)_{j}$ an orthonormal basis $\left(u_{j}(\epsilon)\right)_{j}$ of the direct sum of eigenspaces associated to the $\lambda_{0}$-group. This method allows us to compute the asymptotic expansion of these functions. 
1220 The integral equation methods for eigenvalue problems

\section{References}

[1] M. Abramowitz and I. A. Stegun, Handbook of Mathematical Functions with Formulas, Graphs, and Mathematical Tables, National Bureau of Standards Applied Mathematics Series, vol. 55, US Government Printing Office, Washington, DC, 1964.

[2] H. Ammari and A. Khelifi, Electromagnetic scattering by small dielectric inhomogeneities, J. Math. Pures Appl. (9) 82 (2003), no. 7, 749-842.

[3] O. P. Bruno and F. Reitich, Solution of a boundary value problem for the Helmholtz equation via variation of the boundary into the complex domain, Proc. Roy. Soc. Edinburgh Sect. A 122 (1992), no. 3-4, 317-340.

[4] - Boundary-variation solution of eigenvalue problems for elliptic operators, J. Fourier Anal. Appl. 7 (2001), no. 2, 169-187.

[5] D. L. Colton and R. Kress, Integral Equation Methods in Scattering Theory, Pure and Applied Mathematics (New York), John Wiley \& Sons, New York, 1983.

[6] I. C. Gohberg and E. I. Sigal, An operator generalization of the logarithmic residue theorem and Rouchés theorem, Mat. Sb. (N.S.) 84(126) (1971), 607-629 (Russian).

[7] T. Kato, Perturbation Theory for Linear Operators, 2nd ed., Springer, Berlin, 1980.

[8] M. V. Keldyš, The completeness of eigenfunctions of certain classes of nonselfadjoint linear operators, Uspehi Mat. Nauk 26 (1971), no. 4(160), 15-41 (Russian), English translation: Russ. Math. Surv. 26 (1971), no. 4, 15-44.

[9] P. D. Lamberti and M. Lanza de Cristoforis, An analyticity result for the dependence of multiple eigenvalues and eigenspaces of the Laplace operator upon perturbation of the domain, Glasg. Math. J. 44 (2002), no. 1, 29-43.

[10] R. Mennicken and M. Möller, Root functions, eigenvectors, associated vectors and the inverse of a holomorphic operator function, Arch. Math. (Basel) 42 (1984), no. 5, 455-463.

[11] R. F. Millar, On the Rayleigh assumption in scattering by a periodic surface. II, Proc. Cambridge Philos. Soc. 69 (1971), 217-225.

[12] J.-C. Nédélec, Acoustic and Electromagnetic Equations. Integral Representations for Harmonic Problems, Applied Mathematical Sciences, vol. 144, Springer, New York, 2001.

[13] F. Rellich, Perturbation Theory of Eigenvalue Problems, Gordon and Breach Science Publishers, New York, 1969.

[14] B. Simon, Fifty years of eigenvalue perturbation theory, Bull. Amer. Math. Soc. (N.S.) 24 (1991), no. 2, 303-319.

[15] S. Steinberg, Meromorphic families of compact operators, Arch. Rational Mech. Anal. 31 (1968/1969), 372-379.

[16] M. E. Taylor, Partial Differential Equations. II. Qualitative Studies of Linear Equations, Applied Mathematical Sciences, vol. 116, Springer, New York, 1996.

Abdessatar Khelifi: Centre de Mathématiques Appliquées (CMAP), École Polytechnique, 91128 Palaiseau cedex, France

E-mail address: khelifi@cmapx.polytechnique.fr 


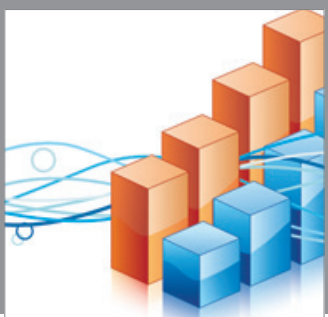

Advances in

Operations Research

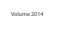

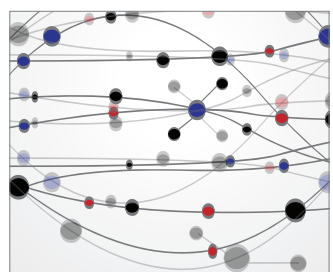

\section{The Scientific} World Journal
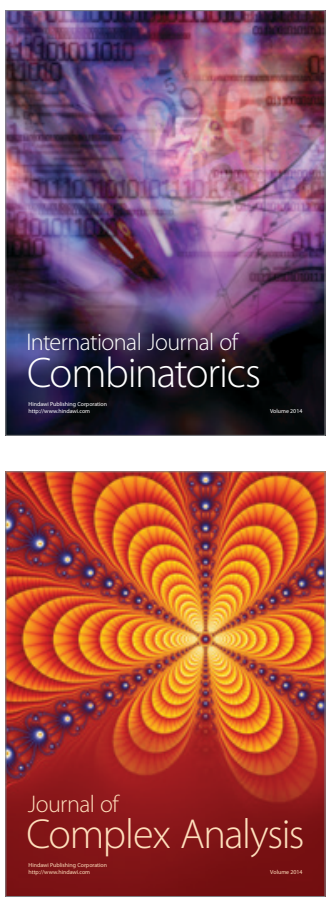

International Journal of

Mathematics and

Mathematical

Sciences
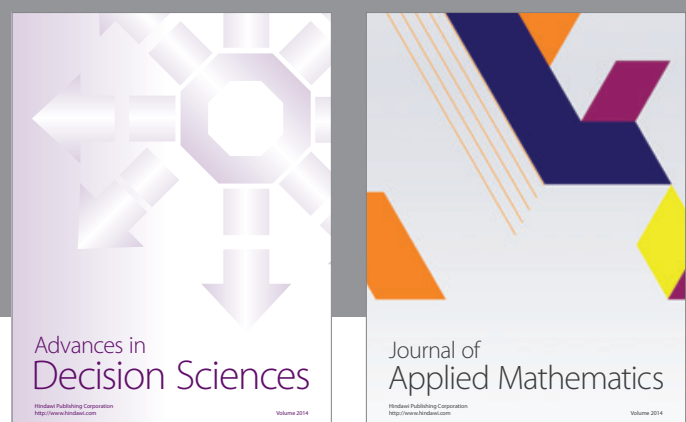

Journal of

Applied Mathematics
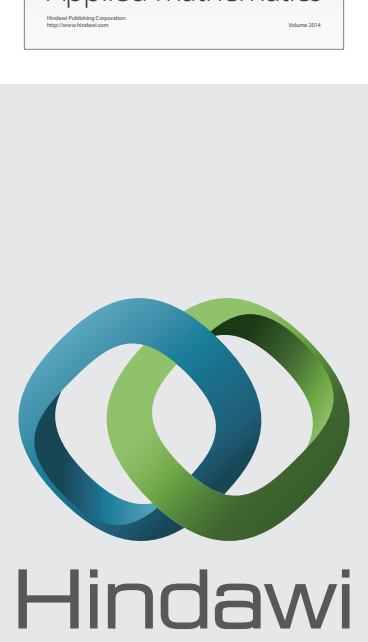

Submit your manuscripts at http://www.hindawi.com
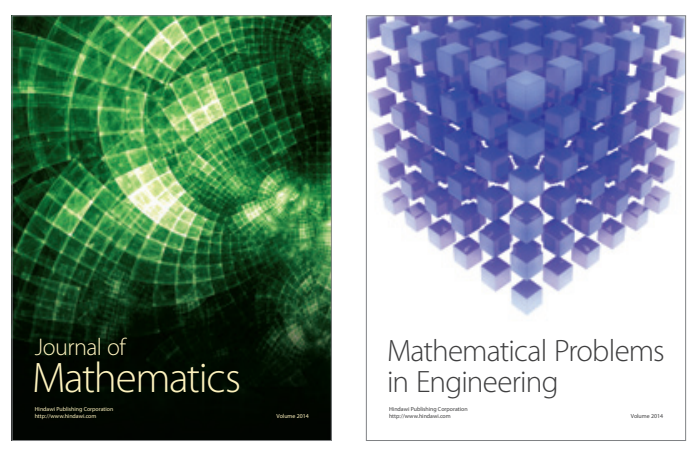

Mathematical Problems in Engineering
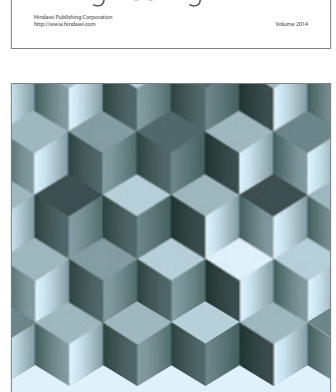

Journal of

Function Spaces
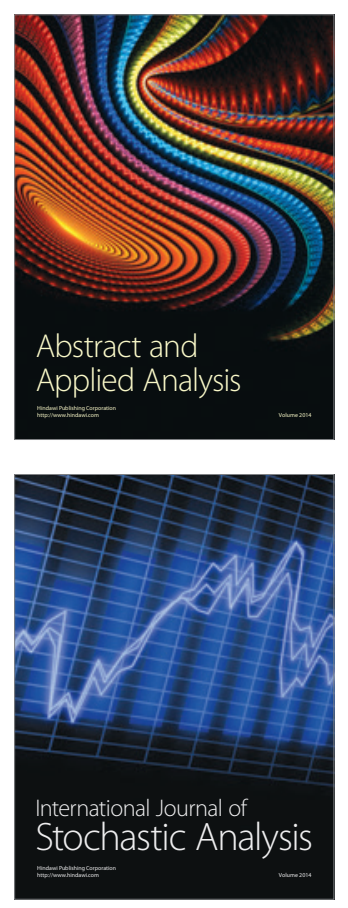

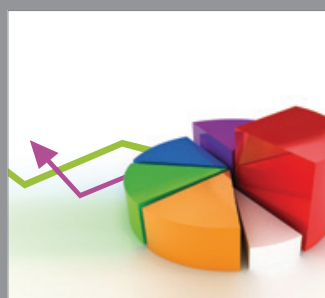

ournal of

Probability and Statistics

Promensencen
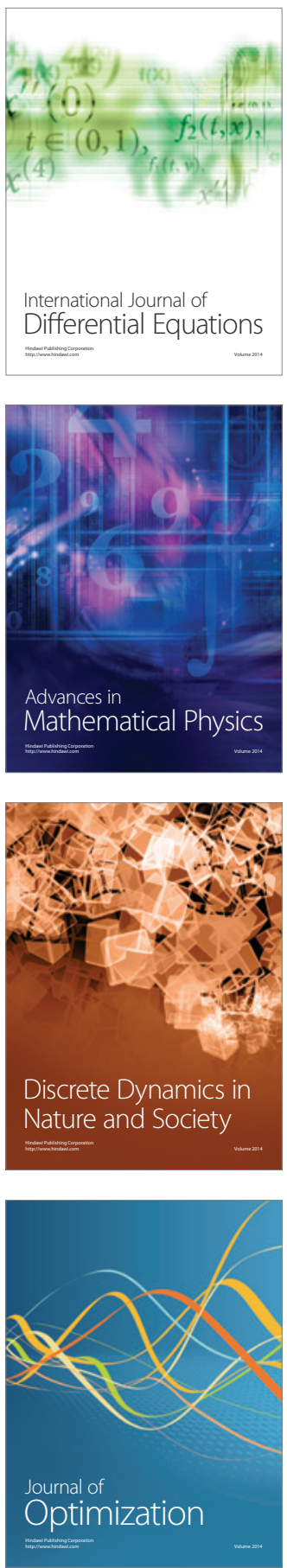